\title{
Relative Positioning and Velocity Estimation Using V2V Delay and Doppler Information
}

\author{
Michael Walter, German Aerospace Center (DLR) \\ Armin Dammann, German Aerospace Center (DLR)
}

\begin{abstract}
BIOGRAPHY
Michael Walter received the Dipl.-Ing. (M.Sc.) and Dr.-Ing. (PhD) degree in electrical engineering from Ulm University, Germany, in 2008 and 2015. Since 2009 he has been a member of the scientific staff at the Institute of Communications and Navigation at the German Aerospace Center (DLR) in Oberpfaffenhofen, Germany, where he gained extensive experience in organizing and conducting numerous measurement campaigns for vehicular and aeronautical research. His research interests include wireless communications, modeling of mobile-to-mobile channels and channel coding. Dr. Walter was the recipient of the IEEE/AIAA David Lubkowski Memorial for Advancement in Digital Avionics Best Paper Award of the 29th DASC in 2011.
\end{abstract}

Armin Dammann studied electrical engineering at the University of Ulm, Germany, with main topic information- and microwavetechnology. He received the Dipl.-Ing. and Dr.-Ing. (PhD) degree in 1997 and 2005 respectively, both from the University of Ulm. In 1997 Armin Dammann joined the Institute of Communications and Navigation of the German Aerospace Center (DLR). Since 2005 he is head of the Mobile Radio Transmission research Group. His research focus currently includes synchronization/positioning for terrestrial OFDM systems.

\section{ABSTRACT}

In this paper, we investigate a relative positioning technique using the vehicle-to-vehicle communication signal. Specifically, we use the delay and Doppler information provided by the channel impulse response. Since the geometry of the vehicles has a direct impact on the delay and Doppler frequency, it is possible to deduce the relative positions of the vehicles. This particular relationship can be exploited by using prolate spheroidal coordinates. By doing an algebraic analysis in this coordinate system closed form solutions for delay and Doppler frequency can be derived. The delay and Doppler information is then used to determine the relative position similar to using the bearing and distance to a lighthouse in marine navigation or distance measuring equipment (DME) and VHF omnidirectional radio range (VOR) in aeronautical navigation.

\section{INTRODUCTION}

The estimation of the relative positions and velocities of transmitter and receiver is important in several wireless applications, like resource management, adaptive modulation and coding, channel assignment, channel estimation, and localization. According to [1] the velocity estimation techniques can be divided into four categories: crossing-based, covariance-based; power spectrum-based; and maximum likelihood-based methods. The absolute value of the velocity with only the transmitter moving is presented in [2]. The authors use a covariance-based algorithm to estimate the speed of the mobile receiver. Another covariance-based method in estimating the maximum Doppler and thus the absolute value of the velocity is shown in [3]. A power spectrum based method is presented in [4], where the absolute value of the velocity of a mobile receiver is estimated. The first publication estimating the transmitter and receiver velocity jointly with the relative direction of movement is given in [1] based on the crossing method for the velocity estimation and correlation based for the direction estimation, i.e., usage of stochastic operators. In [5] the absolute values of transmitter and receiver velocity are estimated based on the maximum likelihood approach. Our novel approach takes into account the information of the instantaneous delay-dependent Doppler power spectrum close to the line-of-sight and far away from it. Since the limiting frequencies are dependent on the relative movement and position, i.e., the geometry, between the vehicles, the velocity components relative to the line-of-sight can be deduced. With this knowledge the angle between the velocity vector of the vehicle and the line-of-sight (LOS) is determined. If 
the line-of-sight is available, the relative positioning is done by angle (using the Doppler spectrum) and distance (using range or pseudo-ranging information) estimation. This approach is similar to estimating the bearing and distance to a lighthouse in marine navigation or DME and VOR in aeronautical navigation. The difference is that both transmitter (Tx) and receiver $(\mathrm{Rx})$ are moving during the velocity and position estimation process. In contrast to the stochastic methods mentioned above, we use the time-variant, distance-dependent relationship between the vehicle geometry and Doppler spectrum to deduce the velocity components and the relative position. Specifically, the Doppler spectrum close to the line of sight (LOS) enables us to estimate the orientation of the velocity vector components relative to the LOS instead of the magnitude of the velocity vectors only. Furthermore, the method does not depend on the particular stochastic distribution of the scatterers as long as scattering from all directions can be observed. Our approach, thus, ensures a time-variant estimation of the velocity vector components and the relative positions between two vehicles. Usually, a time-invariant, narrow-band model is used when estimating the Doppler frequency from the Doppler spectrum. In this approach, however, we use the time-variant, distance-dependent Doppler spectrum, since the limiting frequencies are dependent on the distance for vehicle-to-vehicle (V2V) channels. Closed-form solutions exist for delays close to the LOS and very large delays. A co-moving coordinate system with prolate spheroidal coordinates [6] allows for an adequate description of the V2V communication scenario. In this particular coordinate system the delay coordinate refers to a constant delay between transmitter, scatterer, and receiver. We use the Doppler spectrum at the LOS and in direct vicinity of the LOS. Additionally, it might be necessary to use the Doppler spectrum for large delays of the scatterers to estimate the orthogonal component relative to the LOS of the other vehicle's velocity vector. For the relative positioning, however, the Doppler spectrum very close to the line-of-sight is sufficient, which is a very important fact, since most of the scattering in $\mathrm{V} 2 \mathrm{~V}$ communication occurs close to the vehicles.

\section{Prolate Spheroidal Coordinate System}

The ability to derive closed-form solution for the Doppler frequencies depends mainly on the adequate selection of an appropriate coordinate system. In the case of V2V communication, the prolate spheroidal coordinate system (PSCS) represents a suitable coordinate system for two center problems as shown in [7]. The PSCS is a three-dimensional, curvilinear, orthogonal coordinate system as shown in Fig. 1. The origin of the coordinate system is by definition always in the middle between Tx an Rx and co-moves with the movement of both. The relationship between Cartesian and prolate spheroidal coordinates is given by [8]

$$
\begin{aligned}
& x=l \sqrt{\left(\xi^{2}-1\right)\left(1-\eta^{2}\right)} \cos (\vartheta) \\
& y=l \sqrt{\left(\xi^{2}-1\right)\left(1-\eta^{2}\right)} \sin (\vartheta) \\
& z=l \xi \eta,
\end{aligned}
$$

with $l$ being the focus distance, i.e. the distance from $\mathrm{Tx}$ and $\mathrm{Rx}$ to the origin, $\xi \in[1, \infty)$, and $\eta \in[-1,1] . \xi=\frac{\tau}{\tau_{\text {los }}}$ represents a normalized delay. The absolute delay is calculated by $\tau=\tau_{\text {los }} \xi=\frac{2 l \xi}{c}$ with $c$ being the speed of light. Isosurfaces of $\xi$ represent prolate spheroidal ellipsoids, whereas values of constant $\eta$ are displayed as hyperboloid surfaces. Geometrically, a fixed $\xi$-coordinate represents locations where the sum of the delays to the foci, i.e., Tx and Rx, is constant. A fixed $\eta$-coordinate describes locations where the difference of the delays to the Tx and Rx is constant. Note that the third orthogonal coordinate in the PSCS constitutes a half-plane as iso-surface. This particular coordinate transform effectively leads to polynomial expressions in the $\xi$ - and $\eta$-coordinates in the Doppler frequency.

The big advantage of the PSCS is that by fixing the $\xi$-coordinate, we automatically obtain a fixed delay. Thus, the delaydependent Doppler probability density function (pdf) becomes only dependent on two coordinates in the PSCS; in a planar geometry this dependency reduces even to a single PSCS coordinate. In contrast, fixing the delay in a Cartesian coordinate system still implies the dependency on all three spatial coordinates $x$ and $y$ and $z$. This makes the resulting expressions more difficult.

The description in the PSCS allows us to express both delay and Doppler frequency in a more compact notation in comparison to Cartesian coordinates. The delay from Tx to an arbitrary scatter $d_{\mathrm{t}}$ and the delay from this scatterer to the Rx $d_{\mathrm{r}}$, can be defined as

$$
\begin{aligned}
d_{\mathrm{t}} & =(\xi+\eta) l, \\
d_{\mathrm{r}} & =(\xi-\eta) l, \\
d_{\mathrm{sc}} & =d_{\mathrm{t}}+d_{\mathrm{r}}=2 \xi l,
\end{aligned}
$$


where the total distance $d_{\mathrm{sc}}$, or the total delay $\tau_{\mathrm{sc}}=\frac{d_{\mathrm{sc}}}{c}$, depend only on the $\xi$-coordinate. As has been derived in [9], the Doppler frequency is calculated as spatial gradient of the Tx and Tx distances $d_{\mathrm{t}}$ and $d_{\mathrm{r}}$ projected onto their velocity vectors. Thus, the Doppler frequency is given by

$$
f_{\mathrm{d}}(\mathbf{x})=\left(\mathbf{v}_{\mathrm{t}}^{\mathrm{T}} \nabla d_{\mathrm{t}}(\mathbf{x})+\mathbf{v}_{\mathrm{r}}^{\mathrm{T}} \nabla d_{\mathrm{r}}(\mathbf{x})\right) \frac{f_{\mathrm{c}}}{c}
$$

As mentioned above a delay-dependent description of the Doppler frequency in Cartesian coordinates depends on the $x-, y$-, and $z$-coordinate. Thus, the calculation of the Doppler pdf becomes very cumbersome. In order to simplify the calculation, we transform the mathematical analysis in the PSCS and express the gradient in (5) in prolate spheroidal coordinates.

Consider an arbitrary scalar function $\Psi(\xi, \eta, \vartheta)$ represented in the PSCS. Its gradient $\nabla \Psi$ is computed according to [10] by

$$
\nabla \Psi(\xi, \eta, \vartheta)=\frac{1}{h_{\xi}} \frac{\partial \Psi}{\partial \xi} \mathbf{e}_{\xi}+\frac{1}{h_{\eta}} \frac{\partial \Psi}{\partial \eta} \mathbf{e}_{\eta}+\frac{1}{h_{\vartheta}} \frac{\partial \Psi}{\partial \vartheta} \mathbf{e}_{\vartheta}
$$

where $\mathbf{e}_{\xi}, \mathbf{e}_{\eta}$, and $\mathbf{e}_{\vartheta}$ are the orthonormal basis vectors of the PSCS. They are obtained by transforming the Cartesian basis vectors $\mathbf{e}_{\mathrm{x}}, \mathbf{e}_{\mathrm{y}}$ and $\mathbf{e}_{\mathrm{z}}$ using the following equations

$$
\mathbf{e}_{\alpha}=\frac{\partial x}{\partial \alpha} \frac{\mathbf{e}_{\mathrm{x}}}{h_{\alpha}}+\frac{\partial y}{\partial \alpha} \frac{\mathbf{e}_{\mathrm{y}}}{h_{\alpha}}+\frac{\partial z}{\partial \alpha} \frac{\mathbf{e}_{\mathrm{z}}}{h_{\alpha}}
$$

where the subscript $\alpha \in\{\xi, \eta, \vartheta\}$. In (6) and (7) the scalars $h_{\xi}, h_{\eta}$, and $h_{\vartheta}$ are called scale factors. They ensure the normalization of the basis vectors in the transformed coordinate system. For orthogonal coordinate systems the scale factors are the roots of the three non-zero elements $h_{i}=\sqrt{g_{i i}}$ of the metric tensor g, see [10] for details. In the PSCS, the scale factors $h_{\alpha}$ are calculated as

$$
h_{\xi}=l \sqrt{\frac{\left(\xi^{2}-\eta^{2}\right)}{\left(\xi^{2}-1\right)}}, \quad h_{\eta}=l \sqrt{\frac{\left(\xi^{2}-\eta^{2}\right)}{\left(1-\eta^{2}\right)}}, \quad h_{\vartheta}=l \sqrt{\left(\xi^{2}-1\right)\left(1-\eta^{2}\right)} .
$$

By transforming (5) into prolate spheroidal coordinates, the Doppler frequency is given by

$$
\begin{aligned}
f_{\mathrm{d}}(t ; \xi, \eta, \vartheta)=\frac{f_{\mathrm{c}}}{c} & \left\{\frac{\sqrt{\left(\xi^{2}-1\right)\left(1-\eta^{2}\right)}}{\xi+\eta}\left(v_{\mathrm{t}}^{x} \cos \vartheta+v_{\mathrm{t}}^{y} \sin \vartheta\right)+\frac{\xi \eta+1}{\xi+\eta} v_{\mathrm{t}}^{z}\right. \\
& \left.+\frac{\sqrt{\left(\xi^{2}-1\right)\left(1-\eta^{2}\right)}}{\xi-\eta}\left(v_{\mathrm{r}}^{x} \cos \vartheta+v_{\mathrm{r}}^{y} \sin \vartheta\right)+\frac{\xi \eta-1}{\xi-\eta} v_{\mathrm{r}}^{z}\right\} .
\end{aligned}
$$

with $\mathbf{v}_{\mathrm{t}}=\left[v_{\mathrm{t}}^{x}, v_{\mathrm{t}}^{z}\right]^{\mathrm{T}}$ being the velocity vector of the $\mathrm{Tx}$ and $\mathbf{v}_{\mathrm{r}}=\left[v_{\mathrm{t}}^{x}, v_{\mathrm{t}}^{z}\right]^{\mathrm{T}}$ being the velocity vector of the Rx, respectively. Note that due to the implicit time-variance of the velocity vectors, the Doppler frequency becomes time-variant which is taken into account by the variable $t$.

\section{Time-Variant, Delay-Dependent Doppler PDF}

In the subsequent algebraic analysis, we reduce the general 3D mobile-to-mobile (M2M) description to a $2 \mathrm{D}$ V2V description, which corresponds to $(\xi, \eta)$ coordinates in the PSCS and $(x, z)$ in the Cartesian coordinate system, see Fig.1. The condition for the coordinate system becomes

$$
y=l \sqrt{\left(\xi^{2}-1\right)\left(1-\eta^{2}\right)} \sin (\vartheta)=0,
$$

which is true for $\vartheta=\{0, \pi\}$. The mathematical derivation relies on the geometric-stochastic description of the vehicular environment to obtain a time-variant joint Doppler delay pdf $p\left(t ; f_{\mathrm{d}}, \xi\right)$. Using the Bayes rule, the joint Doppler delay pdf is calculated as $p\left(t ; f_{\mathrm{d}}, \xi\right)=p(t ; \xi) p\left(t ; f_{\mathrm{d}} \mid \xi\right)$.

Subsequently, we determine the delay-dependent conditional Doppler pdf $p\left(t ; f_{\mathrm{d}} \mid \xi\right)$ in the PSCS. The result is obtained by a coordinate transformation of the scatterer distribution $p(\eta \mid \xi)$ as was shown in [7]; subsequently, we summarize the key steps in computing $p\left(t ; f_{\mathrm{d}} \mid \xi\right)$.

We model the delay-dependent pdf of uniformly distributed scatterers as described in [9]. We fix the $\xi$-coordinate and make the assumption that the scatterer distribution is independent on the absolute time $t$, i.e., $p(t ; \eta \mid \xi)=p(\eta \mid \xi)$. Next, we consider 


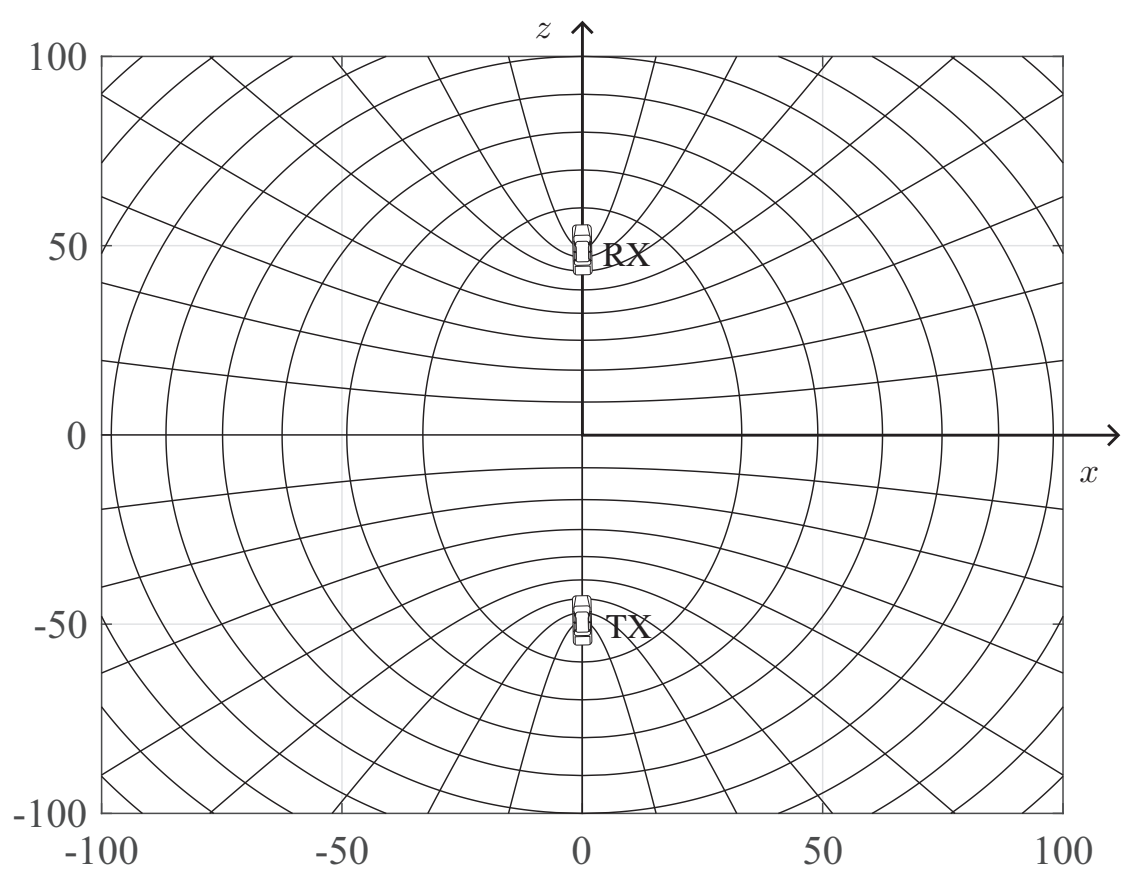

Fig. 1. Prolate spheroidal coordinate system with transmitter and receiver. The relationship to the Cartesian coordinate system is indicated as well.

$\eta \in[-1,1]$ of the half-ellipse that specifies a scatterer lying on it. For a fixed $\xi$, it can be shown [9], [11] that the conditional pdf $p(\eta \mid \xi)$ is computed by applying standard rules of probability density transformations [12] as

$$
p(\eta \mid \xi)=\frac{1}{2} p(\eta, \vartheta=0 \mid \xi)+\frac{1}{2} p(\eta, \vartheta=\pi \mid \xi)=\left.\frac{1}{4 E\left(\frac{1}{\xi^{2}}\right)} \frac{\sqrt{1-\frac{\eta^{2}}{\xi^{2}}}}{\sqrt{1-\eta^{2}}}\right|_{\vartheta=0}+\left.\frac{1}{4 E\left(\frac{1}{\xi^{2}}\right)} \frac{\sqrt{1-\frac{\eta^{2}}{\xi^{2}}}}{\sqrt{1-\eta^{2}}}\right|_{\vartheta=\pi}
$$

where $E\left(\frac{1}{\xi^{2}}\right):=\int_{0}^{1} \sqrt{\frac{1-\frac{\eta^{2}}{\xi^{2}}}{1-\eta^{2}}} \mathrm{~d} \eta$ is the complete elliptic integral of the second kind.

Now we compute the time-variant, delay-dependent Doppler pdf as

$$
p\left(t ; f_{\mathrm{d}} \mid \xi\right)=\left.\sum_{\eta^{\prime} \in\left\{\mathcal{F}^{-1}\left(f_{\mathrm{d}}\right)\right\}} \frac{p(\eta, \vartheta=0 \mid \xi)}{\left|\frac{\partial f_{\mathrm{d}}(t ; \eta, \vartheta=0 \mid \xi)}{\partial \eta}\right|}\right|_{\eta=\eta^{\prime}}+\left.\sum_{\eta^{\prime} \in\left\{\mathcal{F}^{-1}\left(f_{\mathrm{d}}\right)\right\}} \frac{p(\eta, \vartheta=\pi \mid \xi)}{\left|\frac{\partial f_{\mathrm{d}}(t ; \eta, \vartheta=\pi \mid \xi)}{\partial \eta}\right|}\right|_{\eta=\eta^{\prime}}
$$

with the Doppler frequency $f_{\mathrm{d}}(t ; \xi, \eta, \vartheta)$ being computed according to (9). The sum in (11) accounts for the fact that one specific Doppler frequency can be caused by several values of $\eta$, which means that the Doppler frequency is a surjective function. More details of the transformation of the pdfs is found in [9].

\section{Calculation of the Poles of the Time-Variant, Delay-Dependent Doppler Spectrum for Single- BOUNCE SCATTERING}

Usually, a time-invariant, narrow-band model is used when estimating the Doppler frequency from the Doppler spectrum. In our approach, however, we use the time-variant, delay-dependent Doppler spectrum, since the poles are dependent on the delay for mobile-to-mobile channels. In this case closed-form solutions exist for delays close to the LOS, i.e., $\xi=\frac{\tau}{\tau_{105}}=1$ and very large delays, i.e., $\xi=\frac{\tau}{\tau_{\text {los }}} \rightarrow \infty$. According to [13] the poles in the Doppler spectrum can be described by a sixth order polynomial. The structure of the polynomial is quite complex and therefore we only consider several special cases, where compact solutions can be determined.

Thus, we evaluate the poles in the Doppler spectrum at the LOS, i.e., $\xi=1$ and in direct vicinity of the LOS, i.e., $\xi=1+\epsilon$ with $\epsilon>0$ being small. Additionally, it might be necessary to use the Doppler spectrum for large delays of the scatterers 


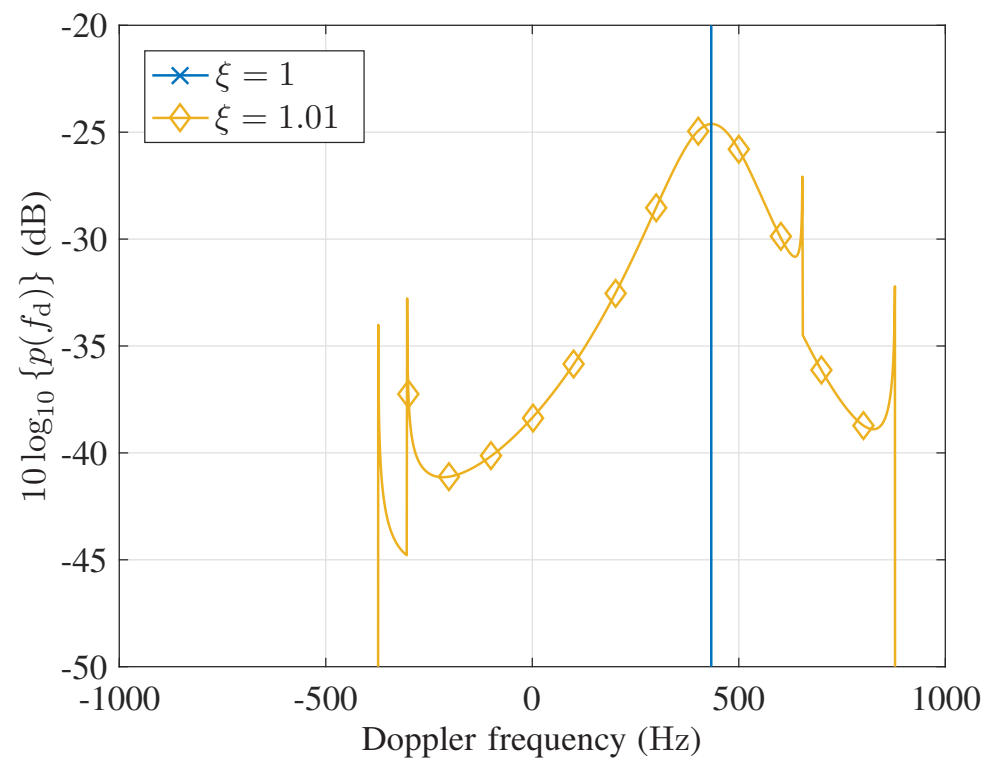

Fig. 2. Doppler spectrum $p\left(t^{*} ; f_{\mathrm{d}} \mid \xi=1.01\right)$ for $\mathbf{v}_{\mathrm{t}}\left(t^{*}\right)=[120,0,-30]^{\mathrm{T}} \mathrm{km} / \mathrm{h}$ and $\mathbf{v}_{\mathrm{r}}\left(t^{*}\right)=[-90,0,60]^{\mathrm{T}} \mathrm{km} / \mathrm{h}$ with isotropic scattering and arbitrary time instant $t^{*}$. Corner frequencies: $f_{\mathrm{b} 1}\left(t^{*}\right)=884 \mathrm{~Hz}, f_{\mathrm{b} 2}\left(t^{*}\right)=665 \mathrm{~Hz}, f_{\mathrm{los}}\left(t^{*}\right)=433 \mathrm{~Hz}, f_{\mathrm{b} 3}\left(t^{*}\right)=-307 \mathrm{~Hz}$, and $f_{\mathrm{b} 4}=-376 \mathrm{~Hz}$.

$(\xi \rightarrow \infty)$ to estimate the sign of the $x$-component of the other vehicle's velocity vector. For the relative positioning, however, the Doppler spectrum very close to the line-of-sight is sufficient.

We introduce the following equations from [13] that are used to determine the velocity components of both transmitter and receiver.

$$
\begin{aligned}
\xi=1+\epsilon: & f_{\mathrm{b} 1,2}(t)=\left( \pm\left\|\mathbf{v}_{\mathrm{t}}(t)\right\|-v_{\mathrm{r}}^{z}(t)\right) \frac{f_{\mathrm{c}}}{c} \\
\xi=1: \quad f_{\mathrm{los}}(t) & =\left(v_{\mathrm{t}}^{z}(t)-v_{\mathrm{r}}^{z}(t)\right) \frac{f_{\mathrm{c}}}{c} \\
\xi=1+\epsilon: & f_{\mathrm{b} 3,4}(t)=\left( \pm\left\|\mathbf{v}_{\mathrm{r}}(t)\right\|+v_{\mathrm{t}}^{z}(t)\right) \frac{f_{\mathrm{c}}}{c} \\
\xi \rightarrow \infty: & f_{\mathrm{e} 1,2}(t)= \pm\left(\left\|\mathbf{v}_{\mathrm{t}}(t)+\mathbf{v}_{\mathrm{r}}(t)\right\|\right) \frac{f_{\mathrm{c}}}{c}
\end{aligned}
$$

We observe the left hand of the equation system in the delay-dependent Doppler spectrum and then infer from it the right hand of the equation system and solve for its unknowns. As can be seen both in (12) and (14), the z-component of the velocity vector is present. Therefore, it becomes possible to calculate both components of the velocity vectors that are parallel to the connecting line between the vehicles. By our definition the Doppler frequencies $f_{\mathrm{b} i}$ are the corner frequency close to the line-of-sight and $f_{\mathrm{e} j}$ are the corner frequencies for large delays. Furthermore, the Doppler frequency of the line-of-sight $f_{\text {los }}$ is needed. In the general case, we obtain four equations for four unknown velocity vector components. Since the magnitude of the velocity vector appears in all, but the line-of-sight equation, the equations are ambiguous in the $x$-component.

The results are valid for arbitrary scatterer distributions, since poles are due to physical limitations, i.e., speed of the vehicles. They are thus determined by the velocity vectors of the vehicles and not the scatterer distribution. Due to this fact our approach is very general and valid for arbitrary Doppler spectra generated by single-bounce scattering.

\section{A. Number of Poles of the Doppler Spectrum close to the Line-of-Sight}

For a fixed, but arbitrary time $t=t^{*}$, the calculation of the poles of the Doppler spectrum can be split in the following cases: 


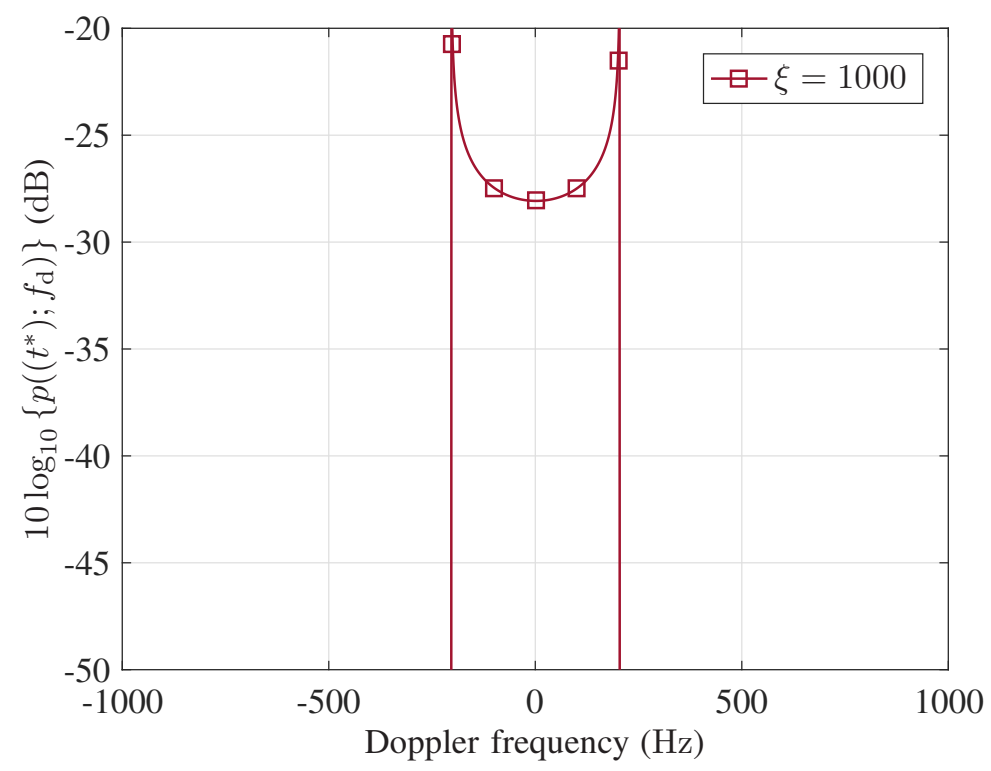

Fig. 3. Doppler spectrum $p\left(t^{*} ; f_{\mathrm{d}} \mid \xi=1000\right)$ for $\mathbf{v}_{\mathrm{t}}\left(t^{*}\right)=[120,0,-30]^{\mathrm{T}} \mathrm{km} / \mathrm{h}$ and $\mathbf{v}_{\mathrm{r}}\left(t^{*}\right)=[-90,0,60]^{\mathrm{T}} \mathrm{km} / \mathrm{h}$ with isotropic scattering. Corner frequencies: $f_{\mathrm{e} 1,2}\left(t^{*}\right)= \pm 204 \mathrm{~Hz}$.

- Two poles:

$$
\begin{aligned}
v_{\mathrm{t}}^{z}\left(t^{*}\right) & =-v_{\mathrm{r}}^{z}\left(t^{*}\right), \quad v_{\mathrm{t}}^{x}\left(t^{*}\right)=v_{\mathrm{r}}^{x}\left(t^{*}\right)=0 \\
f_{b 1}\left(t^{*}\right) & =2 v_{\mathrm{t}}^{z}\left(t^{*}\right) \frac{f_{\mathrm{c}}}{c}, \quad f_{b 2}\left(t^{*}\right)=f_{\text {los }}\left(t^{*}\right)=0
\end{aligned}
$$

- Three poles:

$$
\begin{gathered}
v_{\mathrm{t}}^{z}\left(t^{*}\right)=v_{\mathrm{r}}^{z}\left(t^{*}\right), \quad v_{\mathrm{t}}^{x}\left(t^{*}\right)=v_{\mathrm{r}}^{x}\left(t^{*}\right)=0, \quad f_{b 1}\left(t^{*}\right)=\left(-v_{\mathrm{t}}^{z}\left(t^{*}\right)-v_{\mathrm{r}}^{z}\left(t^{*}\right)\right) \frac{f_{\mathrm{c}}}{c} \\
f_{b 2}\left(t^{*}\right)=f_{\mathrm{los}}\left(t^{*}\right)=\left(v_{\mathrm{t}}^{z}\left(t^{*}\right)-v_{\mathrm{r}}^{z}\left(t^{*}\right)\right) \frac{f_{\mathrm{c}}}{c}, \quad f_{b 3}\left(t^{*}\right)=\left(v_{\mathrm{t}}^{z}\left(t^{*}\right)+v_{\mathrm{r}}^{z}\left(t^{*}\right)\right) \frac{f_{\mathrm{c}}}{c} \\
\text { or } \\
v_{\mathrm{t}}^{x}\left(t^{*}\right)=\left|v_{\mathrm{r}}^{x}\left(t^{*}\right)\right|, \quad v_{\mathrm{t}}^{z}\left(t^{*}\right)=v_{\mathrm{r}}^{z}\left(t^{*}\right)=0 \\
f_{b 1,2}\left(t^{*}\right)= \pm v_{\mathrm{t}}^{x}\left(t^{*}\right) \frac{f_{\mathrm{c}}}{c}, \quad f_{\mathrm{los}}\left(t^{*}\right)=0
\end{gathered}
$$

- Four poles:

$$
\begin{gathered}
v_{\mathrm{r}}^{x}\left(t^{*}\right)=0, \quad v_{\mathrm{t}}^{x}\left(t^{*}\right) \neq 0, \quad v_{\mathrm{t}}^{z}\left(t^{*}\right) \neq 0, \quad v_{\mathrm{r}}^{z}\left(t^{*}\right) \neq 0, \quad f_{b 1,2}\left(t^{*}\right)=\left( \pm\left\|\mathbf{v}_{\mathrm{t}}\left(t^{*}\right)\right\|-v_{\mathrm{r}}^{z}\left(t^{*}\right)\right) \frac{f_{\mathrm{c}}}{c} \\
f_{b 3}\left(t^{*}\right)=f_{\text {los }}=\left(v_{\mathrm{t}}^{z}\left(t^{*}\right)-v_{\mathrm{r}}^{z}\left(t^{*}\right)\right) \frac{f_{\mathrm{c}}}{c}, \quad f_{b 4}\left(t^{*}\right)=\left(v_{\mathrm{t}}^{z}\left(t^{*}\right)+v_{\mathrm{r}}^{z}\left(t^{*}\right)\right) \frac{f_{\mathrm{c}}}{c} \\
\text { or } \\
v_{\mathrm{t}}^{x}\left(t^{*}\right)=0, \quad v_{\mathrm{r}}^{x}\left(t^{*}\right) \neq 0, \quad v_{\mathrm{t}}^{z}\left(t^{*}\right) \neq 0, \quad v_{\mathrm{r}}^{z}\left(t^{*}\right) \neq 0, \quad f_{b 1,2}\left(t^{*}\right)=\left( \pm\left\|\mathbf{v}_{\mathrm{r}}\left(t^{*}\right)\right\|+v_{\mathrm{t}}^{z}\left(t^{*}\right)\right) \frac{f_{\mathrm{c}}}{c} \\
f_{b 3}\left(t^{*}\right)=f_{\text {los }}\left(t^{*}\right)=\left(v_{\mathrm{t}}^{z}\left(t^{*}\right)-v_{\mathrm{r}}^{z}\left(t^{*}\right)\right) \frac{f_{\mathrm{c}}}{c}, \quad f_{b 4}\left(t^{*}\right)=\left(-v_{\mathrm{t}}^{z}\left(t^{*}\right)-v_{\mathrm{r}}^{z}\left(t^{*}\right)\right) \frac{f_{\mathrm{c}}}{c}
\end{gathered}
$$

- Five poles in the general case: non-simplified versions of (12)-(15) are needed.

The Algorithm 1 is used to determine the velocity components and perform the relative positioning task. We hereby assume the magnitude of the own velocity vector $\left\|\mathbf{v}_{\mathrm{t}}\left(t^{*}\right)\right\|$ is known by an odometer for example. 


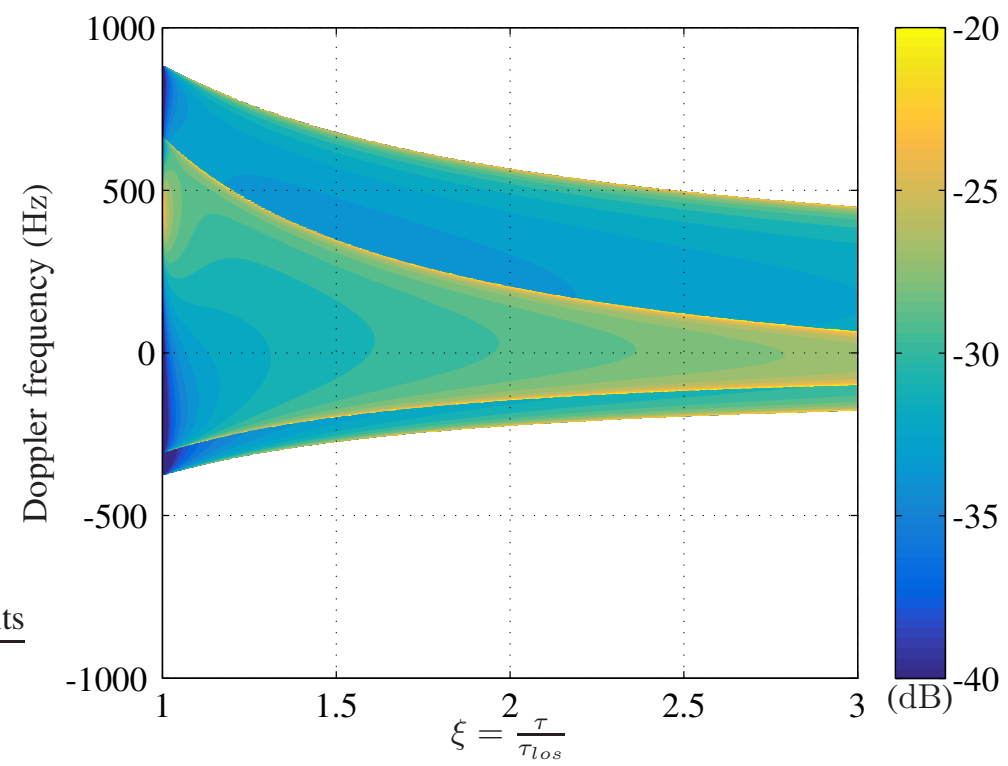

Fig. 4. Delay-dependent Doppler spectrum $p\left(t^{*} ; f_{\mathrm{d}} \mid \xi\right)$ for $\mathbf{v}_{\mathrm{t}}\left(t^{*}\right)=[120,0,30]^{\mathrm{T}} \mathrm{km} / \mathrm{h}$ and $\mathbf{v}_{\mathrm{r}}\left(t^{*}\right)=[-90,0,-60]^{\mathrm{T}} \mathrm{km} / \mathrm{h}$.
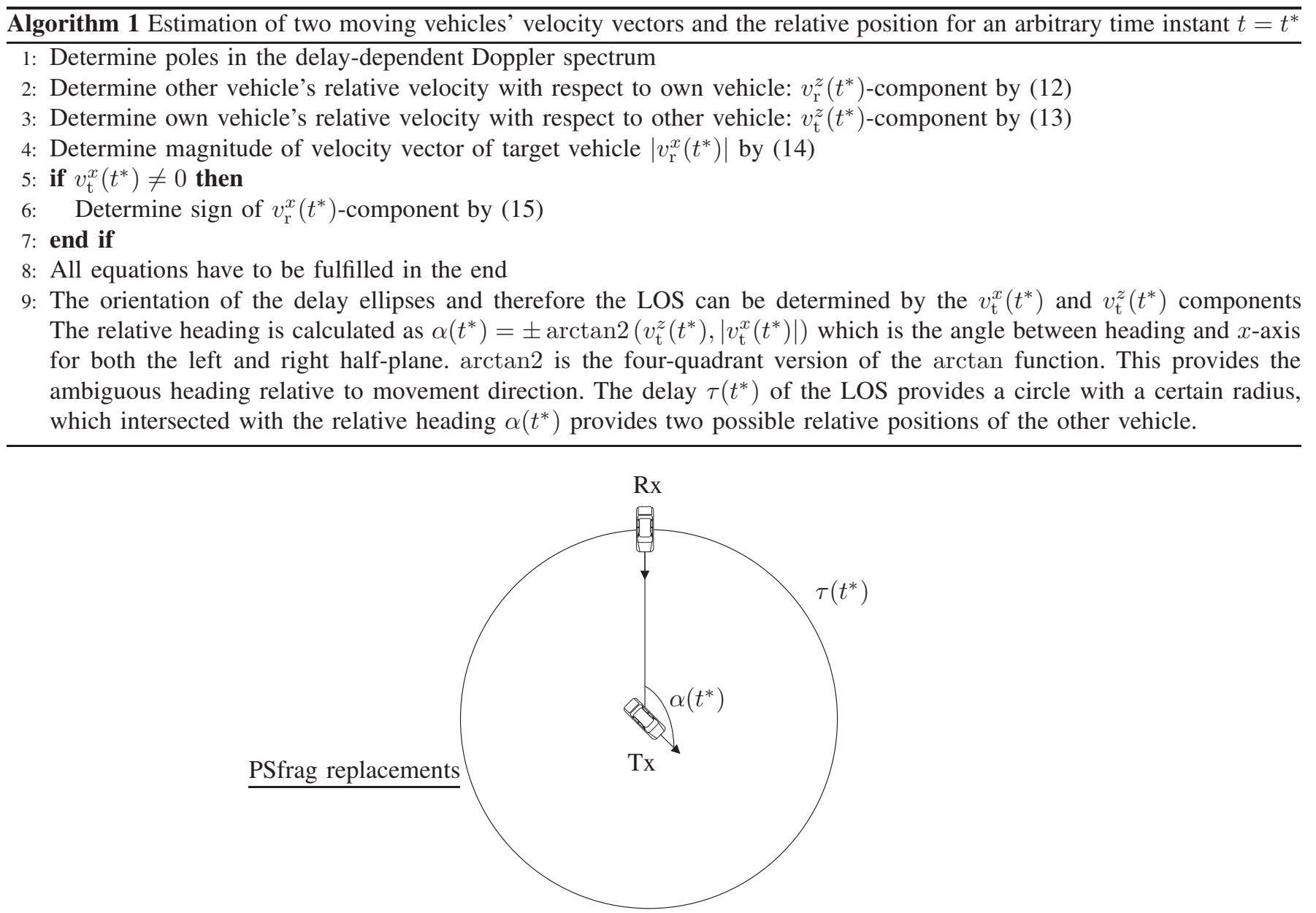

Fig. 5. Relative positioning with the help of the calculated relative heading $\alpha\left(t^{*}\right)$ and the line-of-sight delay $\tau\left(t^{*}\right)$. The Tx vehicle is the ego vehicle at arbitrary time instant $t=t^{*}$ 


\section{RESULTS}

In the selected exemplary case the transmitter vehicle drives with a velocity vector of $\mathbf{v}_{\mathrm{t}}\left(t^{*}\right)=[120,0,-30]^{\mathrm{T}} \mathrm{km} / \mathrm{h}$ and the receiver with a velocity vector of $\mathbf{v}_{\mathrm{r}}\left(t^{*}\right)=[-90,0,60]^{\mathrm{T}} \mathrm{km} / \mathrm{h}$. Isotropic scattering is assumed. The resulting poles in the spectrum are at $f_{\mathrm{b} 1}\left(t^{*}\right)=884 \mathrm{~Hz}, f_{\mathrm{b} 2}\left(t^{*}\right)=665 \mathrm{~Hz}, f_{\mathrm{los}}\left(t^{*}\right)=433 \mathrm{~Hz}, f_{\mathrm{b} 3}\left(t^{*}\right)=-307 \mathrm{~Hz}, f_{\mathrm{b} 4}\left(t^{*}\right)=-376 \mathrm{~Hz}$, and $f_{\mathrm{e} 1,2}\left(t^{*}\right)= \pm 204 \mathrm{~Hz}$. With the knowledge of those poles, it becomes possible with (12)-(15) to determine the components of the velocity vector and perform relative positioning between the vehicles. By using Algorithm 1, the velocity components can be correctly estimated.

The estimation of the relative positioning together with the estimation of the velocity components can be used as additional functionality for vehicle-to-vehicle communications systems. It enables the vehicle to get a better situational awareness. As Fig. 5 shows the angle $\alpha\left(t^{*}\right)$ is estimated by the velocity components. The delay to the other vehicle is obtained by using the propagation time of the line-of-sight component, which provides us with a delay circle around our ego vehicle. Subsequently, the circle is intersected with the radial of the angle $\alpha\left(t^{*}\right)$ and we obtain one intersection point in each half plane, where the other vehicle could be located. The determination of the relative position is thus similar to estimate the angle and distance to a lighthouse in marine navigation or DME and VOR in aeronautical navigation with the difference that we estimate the velocity components and thus the angle from the delay-dependent Doppler spectrum. This method becomes possible by using closed-form equations for the Doppler spectrum for single-bounce scattering.

\section{CONCLUSION}

In this paper we have derived an algorithm to perform relative positioning and velocity estimation by exploiting the delay and Doppler information from the vehicle-to-vehicle communication signal. Since the delay-dependent Doppler spectrum depends on the geometry of the vehicles and its velocity vectors, the angle to the other vehicle can be determined. The calculation becomes possible by using closed-form solutions for the poles of the Doppler spectrum for delays close to the line-of-sight and for very large delays.

\section{REFERENCES}

[1] A. G. Zajić, "Estimation of mobile velocities and direction of movement in mobile-to-mobile wireless fading channels," IEEE Transactions on Vehicular Technology, vol. 61, no. 1, pp. 130-139, Jan. 2012.

[2] C. Tepedelenlioglu and G. B. Giannakis, "On velocity estimation and correlation properties of narrow-band mobile communication channels," IEEE Transactions on Vehicular Technology, vol. 50, no. 4, pp. 1039-1052, Jul. 2001.

[3] A. Abdi, H. Zhang, and C. Tepedelenlioglu, "A Unified Approach to the Performance Analysis of Speed-Estimation Techniques in Mobile Communication," IEEE Transactions on Communications, vol. 56, no. 1, pp. 126-135, Jan. 2008.

[4] S. Mohanty, "VEPSD: a novel velocity estimation algorithm for next-generation wireless systems," IEEE Transactions on Wireless Communications, vol. 4, no. 6, pp. 2655-2660, Nov. 2005.

[5] R. O. Adeogun, P. D. Teal, and P. A. Dmochowski, "Extrapolation of MIMO Mobile-to-Mobile Wireless Channels Using Parametric-Model-Based Prediction," IEEE Transactions on Vehicular Technology, vol. 64, no. 10, pp. 4487-4498, Oct. 2015.

[6] C. Flammer, Spheroidal Wave Functions. Stanford University Press, 1957.

[7] M. Walter, D. Shutin, and U.-C. Fiebig, "Prolate Spheroidal Coordinates for Modeling Mobile-to-Mobile Channels," IEEE Antennas and Wireless Propagation Letters, vol. 14, pp. 155-158, 2015.

[8] M. Abramowitz and I. A. Stegun, Handbook of Mathematical Functions: With Formulas, Graphs, and Mathematical Tables, ser. Applied mathematics series. Dover Publications, 1965.

[9] M. Walter, D. Shutin, and U.-C. Fiebig, "Delay-Dependent Doppler Probability Density Functions for Vehicle-to-Vehicle Scatter Channels," IEEE Transactions on Antennas and Propagation, vol. 62, no. 4, pp. 2238-2249, Apr. 2014.

[10] I. N. Bronstein, K. A. Semendyayev, G. Musiol, and H. Mühlig, Handbook of Mathematics. Springer, 2007.

[11] O. Nørklit and J. B. Andersen, "Diffuse Channel Model and Experimental Results for Array Antennas in Mobile Environments," IEEE Transactions on Antennas and Propagation, vol. 46, no. 6, pp. 834-840, Jun. 1998.

[12] A. Papoulis and S. U. Pillai, Probability, Random Variables, and Stochastic Processes, ser. McGraw-Hill electrical and electronic engineering series. McGraw-Hill, 2002.

[13] M. Walter, D. Shutin, and A. Dammann, "Algebraic analysis of the poles in the Doppler spectrum for vehicle-to-vehicle channels," IEEE Wireless Communications Letters, vol. 7, no. 4, pp. 662-665, Aug. 2018. 